\title{
AFFINE STRUCTURES ON THREE-STEP NILPOTENT LIE ALGEBRAS
}

\section{JOHN SCHEUNEMAN}

ABSTRACT. As part of the investigation of the parallel between solvable Lie theory and the theory of groups of affine motions, it is proved that every three-step nilpotent Lie algebra admits a faithful representation of a certain special kind. It follows immediately that every three-step nilpotent Lie group which is connected, simply connected, and of dimension $n$ admits a representation as a simply transitive group of affine motions of $R^{n}$.

1. Introduction. Denote by $\mathfrak{A}_{n}$ the affine Lie algebra consisting of all $(n+1) \times(n+1)$ matrices $X=\left(\begin{array}{cc}A & v \\ 0 & 0\end{array}\right)$ with bottom row zero and with Lie product $[X, Y]=X Y-Y X$. The column vector $v$ will be called the translation part of $X$ and the $n \times n$ matrix $A$ will be called the homogeneous part. By an affine structure on an $n$-dimensional Lie algebra $L$, we mean a Lie algebra homomorphism $f: L \rightarrow \mathbb{Q}_{n}$ such that the set of translation parts of elements of $f(L)$ equals all of $n$-space. The map $f$ is then a faithful representation of $L$, and the only element of $f(L)$ with zero translation part is zero.

Our interest in the notion of an affine structure has a geometrical source, namely the fact that the Lie algebra of a simply transitive group of affine motions of $R^{n}$ has an affine structure. Such Lie groups can be shown to be solvable, and also, the complete locally affine spaces are known to be closely related to solvmanifolds (see [1]), but the full extent of the parallel between solvable Lie theory and the theory of groups of affine motions is not known. This article is directed at the question of what solvable (especially nilpotent) Lie groups admit representations as simply transitive groups of affine motions of $R^{n}$. It was noted by $\dot{E}$. Cartan that every twostep nilpotent Lie group (connected and simply connected, as usual here)

Received by the editors October 30, 1973.

AMS (MOS) subject classifications (1970). Primary 22E25; Secondary 17 B 30. Key words and phrases. Simply transitive group of affine motions. 
has such a representation. Also, if a nilpotent Lie algebra has a nonsingular derivation, then again the associated Lie group admits such a representation (see [2]). The same is true for nilpotent Lie groups whose Lie algebra is an associative nilpotent algebra made Lie by taking $[X, Y]=$ $X Y-Y X$ (unpublished result of L. Auslander, quoted with permission).

The result of the present article, that each three-step nilpotent Lie algebra has an affine structure, can easily (just exponentiate) be seen to imply that the associated simply connected Lie group can also be made to act as a simply transitive group of affine motions. Although this is again a special case, it is sufficiently general that, taken with what else is known, it leads to the conjecture that all simply connected nilpotent Lie groups have representations as above.

2. The theorem. The result to be proved is that if $L$ is a three-step nilpotent Lie algebra, then $L$ has an affine structure $f$ such that $f(L)$ consists of nilpotent matrices. Let $W=[L,[L, L]]$; choose a complement $V$ to $W$ in $[L, L]$ and a complement $U$ to $[L, L]$ in $L$. Then we have $L=U \oplus V \oplus W$ (as a vector space), and $[U, U] \subset V \oplus W,[U, V] \subset W$, and $[U, W]=[V, V]=[V, W]=[W, W]=0$. Next choose a basis $\left\{w_{1}, \ldots, w_{m}\right.$, $\left.v_{1}, \cdots, v_{n}, u_{1}, \cdots, u_{p}\right\}$ for $L$, where the $w^{\prime} s, v^{\prime} s$, and $u^{\prime} s$ are a basis for $W, V, U$, respectively. Write

$$
\left[u_{i}, u_{j}\right]=\sum_{k} a_{i k j} v_{k}+\sum_{k} c_{i k j} w_{k} \text { and }\left[u_{i}, v_{j}\right]=\sum_{k} b_{i k j} w_{k} .
$$

If we identify elements of $L$ with column vectors and the above basis vectors (in the above order) with the usual basis of the space of column vectors, and then compute the matrices of the adjoint representation, we find the following pattern:

ad $u_{i}=\left(\begin{array}{ccc}0 & B_{i} & C_{i} \\ 0 & 0 & A_{i} \\ 0 & 0 & 0\end{array}\right), \quad$ ad $v_{i}=\left(\begin{array}{ccc}0 & 0 & D_{i} \\ 0 & 0 & 0 \\ 0 & 0 & 0\end{array}\right)$ and $\operatorname{ad} w_{i}=\left(\begin{array}{ccc}0 & 0 & 0 \\ 0 & 0 & 0 \\ 0 & 0 & 0\end{array}\right)$.

The entries in the blocks $A, B, C$ are $a$ 's, $b$ 's, and $c^{\prime}$ 's, respectively; the $k j$ th entry $d_{i k j}$ of $D_{i}$ is $-b_{j k i}$. Notice that

$\left[\operatorname{ad} u_{i}, \operatorname{ad} u_{j}\right]_{=}=\left(\begin{array}{ccc}0 & 0 & B_{i} A_{j}-B_{j} A_{i} \\ 0 & 0 & 0 \\ 0 & 0 & 0\end{array}\right)=\operatorname{ad}\left[u_{i}, u_{j}\right]=\sum_{k} a_{i k j}\left(\begin{array}{ccc}0 & 0 & D_{k} \\ 0 & 0 & 0 \\ 0 & 0 & 0\end{array}\right)$. 
We now obtain a representation of $L$ related to the adjoint representation by weighting the blocks as follows: replace ad $u_{i}$ by

and ad $v_{i}$ by

$$
U_{0 i}=\left(\begin{array}{ccc}
0 & b B_{i} & c C_{i} \\
0 & 0 & a A_{i} \\
0 & 0 & 0
\end{array}\right)
$$

$$
V_{0 i}=\left(\begin{array}{ccc}
0 & 0 & d D_{i} \\
0 & 0 & 0 \\
0 & 0 & 0
\end{array}\right) .
$$

Then the linear map given by $u_{i} \rightarrow U_{0 i}, v_{i} \rightarrow V_{0 i}, w_{i} \rightarrow 0$ will be a representation of $L$ provided the numbers $a, b, c, d$ satisfy the relation $d=a b$.

We may now give an affine structure for $L$ based on the above representation as follows. Let $f: L \rightarrow \mathbb{A}_{n}$ be the linear map defined by

$$
u_{i} \rightarrow U_{i}=\left(\begin{array}{cccc}
0 & b B_{i} & c C_{i} & 0 \\
0 & 0 & a A_{i} & 0 \\
0 & 0 & 0 & u_{i} \\
0 & 0 & 0 & 0
\end{array}\right), \quad v_{i} \rightarrow V_{i}=\left(\begin{array}{cccc}
0 & 0 & a b D_{i} & 0 \\
0 & 0 & 0 & r v_{i} \\
0 & 0 & 0 & 0 \\
0 & 0 & 0 & 0
\end{array}\right) \text {, }
$$

and

$$
w_{i} \rightarrow w_{i}=\left(\begin{array}{cccc}
0 & 0 & 0 & s w_{i} \\
0 & 0 & 0 & 0 \\
0 & 0 & 0 & 0 \\
0 & 0 & 0 & 0
\end{array}\right)
$$

It remains to verify that $a, b, c, r$, and $s$ can be chosen so that $r$ and $s$ are nonzero and $f$ is a homomorphism. First notice that each $\left[V_{i}, V_{j}\right]=0$ and that each $W_{i}$ commutes with all the $U^{\prime} s, V^{\prime} s$ and $W^{\prime} s$, as required. Next, since the homogeneous parts of the $U^{\prime} s, V^{\prime} s$ and the W's have been constructed to give a representation, it suffices now to consider the translation parts of $\left[U_{i}, U_{j}\right]$ and $\left[U_{i}, V_{j}\right]$. The former is

$$
\left(\begin{array}{c}
c C_{i} u_{j}-c C_{j} u_{i} \\
a A_{i} u_{j}-a A_{j} u_{i} \\
0
\end{array}\right)=\left(\begin{array}{c}
2 c \Sigma c_{i k j} w_{k} \\
2 a \Sigma a_{i k j} v_{k} \\
0
\end{array}\right)=\sum a_{i k j}\left(\begin{array}{c}
0 \\
r v_{k} \\
0
\end{array}\right)+\sum c_{i k j}\left(\begin{array}{c}
s w_{k} \\
0 \\
0
\end{array}\right)
$$


provided $s=2 c$ and $r=2 a$. The translation part of $\left[U_{i}, V_{j}\right]$ is

$$
\left(\begin{array}{c}
b r B_{i} v_{j}-a b D_{j} v_{i} \\
0 \\
0
\end{array}\right)=\left(\begin{array}{c}
(b r+a b) \sum b_{i k j} w_{k} \\
0 \\
0
\end{array}\right)=\sum b_{i k j}\left(\begin{array}{c}
s w_{k} \\
0 \\
0
\end{array}\right) \text {, }
$$

provided $s=b r+a b$. Thus $f$ is an affine structure if the weights $a, b, c$, $r$, and $s$ are all nonzero and satisfy the three conditions $r=2 a, s=2 c$, $s=b r+a b$ (Example: $a=b=c=2 / 3$ and $r=s=4 / 3$ ).

Remark. The analogous method of choosing a basis, computing the adjoint representation, and weighting blocks does not work in the four-step nilpotent case, and therefore it fails in the $n$-step case, also, if $n>4$.

\section{REFERENCES}

1. L. Auslander, Structure of complete locally affine manifolds, Topology 3 (1964), suppl. 1, 131-139. MR $28 \# 4463$.

2. J. Scheuneman, Examples of compact locally affine spaces, Bull. Amer. Math. Soc. 77 (1971), 589-592. MR 44 \#7554.

24 SOUTH DUTCHER STREET, IRVINGTON, NEW YORK 10533 